\title{
Correction to: Preparation and characterization of renewable composites from Polylactide and Rice husk for 3D printing applications
}

\author{
Chi-Hui Tsou ${ }^{1,2}$ • Wei-Hua Yao ${ }^{3} \cdot$ Chin-San $\mathrm{Wu}^{4} \cdot$ Chih-Yuan Tsou $^{1} \cdot$ Wei-Song Hung ${ }^{5,6} \cdot$ Jui-Chin Chen $^{3} \cdot$ Jipeng Guo $^{1}$. \\ Shuai Yuan ${ }^{1} \cdot$ Ehua Wen ${ }^{1} \cdot$ Ruo-Yao Wang ${ }^{5} \cdot$ Maw-Cherng Suen $^{7} \cdot$ Shi-Chih Liu $^{7}$ - Manuel Reyes De Guzman ${ }^{1}$
}

Published online: 6 January 2020

(C) The Polymer Society, Taipei 2019

\section{Correction to: Journal of Polymer Research (2019) 26:227} https://doi.org/10.1007/s10965-019-1882-6

The original version of this article unfortunately contained a mistake. The name "Maw-Cheng Sunn" should be corrected to "Maw-Cherng Suen".

The original article has been corrected.

The online version of the original article can be found at https://doi.org/ 10.1007/s10965-019-1882-6

Chi-Hui Tsou mayko0301@hotmail.com

$\triangle$ Manuel Reyes De Guzman manuelrdg@yahoo.com

1 Material Corrosion and Protection Key Laboratory of Sichuan Province, College of Materials Science and Engineering, Sichuan University of Science and Engineering, Zigong 643000, China

2 Department of Materials Science, Chulalongkorn University, Bangkok 10330, Thailand

3 Department of Materials and Textiles, Oriental Institute of Technology, Pan-Chiao 22064, Taiwan, Republic of China

4 Department of Applied Cosmetology, Kao Yuan University, Kaohsiung 82101, Taiwan, Republic of China

5 Department of Materials Science and Engineering, National Taiwan University of Science and Technology, Taipei 10607, Taiwan, Republic of China

$6 \quad$ R\&D Center for Membrane Technology, Department of Chemical Engineering, Chung Yuan University, Chung-Li 32023, Taiwan, Republic of China

7 Department of Fashion Business Administration, Lee-Ming Institute of Technology, Taishan, New Taipei City 24305, Taiwan, Republic of China 